\title{
Error-Mitigated Digital Quantum Simulation
}

\author{
Sam McArdle, ${ }^{*}$ Xiao Yuan, ${ }^{\dagger}$ and Simon Benjamin ${ }^{\ddagger}$ \\ Department of Materials, University of Oxford, Parks Road, Oxford OXI 3PH, United Kingdom
}

(Received 8 August 2018; published 8 May 2019)

\begin{abstract}
Variational algorithms may enable classically intractable simulations on near-future quantum computers. However, their potential is limited by hardware errors. It is therefore crucial to develop efficient ways to mitigate these errors. Here, we propose a stabilizerlike method which enables the detection of up to $60 \%-80 \%$ of depolarizing errors. Our method is suitable for near-term quantum hardware. Simulations show that our method can significantly benefit calculations subject to both stochastic and correlated noise, especially when combined with existing error mitigation techniques.
\end{abstract}

DOI: 10.1103/PhysRevLett.122.180501

Unlike their classical counterparts, quantum computers can efficiently simulate large quantum systems [1-3]. For example, using a quantum computer, we can efficiently find the ground states of systems such as the Fermi-Hubbard model or molecules [4]. Accurately determining the ground states of quantum systems is a first step towards developing new materials [5], more effective medicines [6], and better catalysts [7]. On first inspection, transformative simulations seem tantalizingly close, requiring only around 100 logical qubits [7]. However, the required circuit depth necessitates at least 500000 physical qubits (based on current error rates and fault-tolerance protocols) [7-9], which is many years beyond our current capabilities.

In contrast, hybrid algorithms, such as the variational quantum eigensolver (VQE) [10-12], may not require error correction [13]. These algorithms exchange the long gate sequences described above for a polynomial number of short circuits, which dramatically reduces the coherence time required. While previous small experimental demonstrations of the VQE have shown it to be resilient to systematic errors [14], larger experiments have shown that noise can corrupt results $[15,16]$. This is perhaps unsurprising; depending on the problem tackled, errors can add or remove particles, effectively changing the system being simulated [17].

While error correction is needed to fully suppress these errors, this requires considerable additional resources [18]. Alternatively, error rates can be lowered by improving the hardware directly. However, this is an enormous challenge; decades of research has reduced error rates to $0.1 \%$, but this has not been improved in several years [19-21]. While

Published by the American Physical Society under the terms of the Creative Commons Attribution 4.0 International license. Further distribution of this work must maintain attribution to the author(s) and the published article's title, journal citation, and DOI. efforts to improve error rates continue worldwide, softwarebased methods to effectively reduce error rates are clearly invaluable.

To date, several methods for mitigating errors in near-term quantum hardware have been proposed: the linear $[22,23]$ and exponential [24] extrapolation methods, the quasiprobability method $[23,24]$, and the quantum subspace expansion [25]. Some of these methods have recently been experimentally demonstrated [26-28]. However, these schemes are generally limited to low error rates, where the number of errors expected in the circuit is on the order of unity. There also exist penalty term methods, which drive the calculation towards a state which respects conserved quantities $[12,29,30]$. While such methods mitigate some algorithmic and coherent errors, they cannot mitigate stochastic errors.

Motivated by these limitations, we have developed a new scheme for error mitigation. Our method uses checks on a suitably constructed trial state to filter errors. It can be used in isolation, or combined with previous error mitigation techniques. We numerically demonstrate our method in an electronic structure calculation on the hydrogen molecule. The method is low cost, and suitable for the emerging generation of quantum hardware. Our technique is applicable to calculations of both static properties (such as ground and excited states and vibrational spectra) and dynamical properties (such as time evolved correlation functions). However, herein we focus on the application of our method to the ground state problem, for clarity of exposition.

Conventional VQE.-We can use the $\mathrm{VQE}$ to find the ground state energy of physical Hamiltonians (see Supplemental Material [31]). In molecular simulations, we seek to arrange $N$ electrons among $M$ spin orbitals, such that the energy of the system is minimized. Here, we use the Jordan-Wigner mapping, where each qubit represents an electron spin orbital, the occupation number of which is stored in the $|0\rangle$ or $|1\rangle$ state of the qubit (unoccupied or occupied, respectively). For example, a state describing the hydrogen molecule $\left(\mathrm{H}_{2}\right)$ is 


$$
\left|\psi^{\mathrm{H}_{2}}\right\rangle=\alpha|0101\rangle+\beta|1010\rangle+\gamma|1001\rangle+\delta|0110\rangle,
$$

where $\alpha, \beta, \gamma, \delta$ are complex coefficients. The Hamiltonian can be written as a linear combination of products of Pauli operators, $H=\sum_{i} g_{i} \hat{h}_{i}$, where $g_{i}$ is a scalar coefficient determining the strength of the term. An example of a typical term is $\hat{h}_{i}=X_{0} Y_{1} Y_{2} X_{3}$.

The VQE, as proposed in Ref. [10], augments a small quantum processor with a powerful classical computer. The quantum computer is used for classically intractable state preparation and energy measurement. The state preparation circuit consists of a number of parametrized gates. The circuit used is known as the Ansatz, denoted by $U(\vec{\theta})$. This circuit produces a trial state, $|\psi(\vec{\theta})\rangle$. The values of the parameters and the energy of the state they create are input into a classical optimization algorithm, which seeks the ground state. The energy is calculated by summing the expectation values of each term in the Hamiltonian. To obtain each expectation value, we repeatedly perform the circuit, measure the state produced, and reinitialize.

Stabilizer VQE.-In variational simulations, it is often beneficial to initialize the register in a mean-field state and use a particle number and spin conserving Ansatz [39,40]. This produces states with the correct number of electrons $N$, spin-up electrons $N_{\uparrow}$, and spin-down electrons $N_{\downarrow}$. We refer to these states as "physical" states. As these quantities are conserved, their relevant parity operators are also conserved: $\hat{P}_{N}|\psi\rangle=P_{N}|\psi\rangle=(-1)^{N}|\psi\rangle$ and $\hat{P}_{N_{\uparrow / \downarrow}}|\psi\rangle=$ $P_{N_{\uparrow / \downarrow}}|\psi\rangle=(-1)^{N_{\uparrow / \downarrow}}|\psi\rangle$. This is similar to the concept of stabilizer states used in quantum error correcting codes [41].

There are some Ansätze, such as those suggested in Ref. [40], which are constructed from individual gates which conserve particle number. If a single bit-flip error occurs, it will create or destroy an electron, radically changing the state. For other number and spin conserving Ansätze, like the singlet unitary coupled cluster (UCC) Ansatz (which, in its canonical form [42], is constructed from individual gates which do not necessarily conserve particle number), a single error can propagate and degrade the final state even further. A key concern for the VQE is preserving particle number, as states with electron number far from the true value appear to have a larger energy variance than those with smaller particle number errors [17]. We present below a method of detecting and removing some of these damaging errors, while still retaining the low qubit resources and gate count of the VQE.

In order to detect errors, we introduce an ancilla qubit and use it to perform measurements of the conserved quantities. When deriving error detection rates, we make the following assumptions: (1) errors are symmetric and depolarizing, (2) the error rate is low, such that only one gate malfunctions, (3) the Ansatz circuit is built from individual gates which conserve particle number and spin, and (4) single-qubit gate error rates are negligible compared to 2-qubit gate error rates.

While our method is still applicable under higher noise rates, different noise models, and using other number conserving Ansätze-as shown by our numerical simulations-calculating an analytic bound becomes more difficult without these assumptions.

The simplest check is of the total electron number parity. This procedure is shown in Fig. 1. Each CNOT gate flips the ancilla qubit if the corresponding spin orbital is occupied by an electron. The circuit enables the detection of any error which changes the electron number parity by one. Under the assumptions described above, we are able to detect and remove $53 \%$ of error events (8/15 errors in the depolarizing noise model, as described in the Supplemental Material [31]). In the Supplemental Material we present an alternative circuit for this parity check, which can reduce the impact of qubit readout errors. The qubit readout error rate is currently around 1\% for superconducting qubits [43]. This alternative circuit also makes it possible to combine our method with existing variational algorithms for real [22] and imaginary [44] time evolution.

To increase the proportion of errors detected, we can perform the circuit shown in Fig. 2 which measures both the spin-up parity and spin-down parity. This enables us to detect additional 2-qubit bit-flip errors that we were unable to detect using the single parity check above. We can effectively filter out $66 \%$ of errors, as shown in the Supplemental Material [31].

We can also measure the electron number and spin numbers directly, using an iterative procedure. We first write the electron number in binary. We then use the circuit in Fig. 3 to measure the first bit in $N$, by setting $m=1$. We denote the bit value measured as $N_{1}$. We then repeat the circuit in Fig. 3 to measure the second bit in $N$, by setting $m=2$, and using our measurement of $N_{1}$ in the rotation $\omega_{2}$. In general, we can measure the $m$ th bit of $N$ using the circuit in Fig. 3, constructing $\omega_{m}$ using our measurements of the $m-1$ preceding bits in $N$. When no errors have occurred, the ancilla is in the state

$$
|\phi\rangle=\frac{1}{\sqrt{2}}\left(\left|0_{a}\right\rangle+e^{N_{m} \pi i}\left|1_{a}\right\rangle\right) .
$$

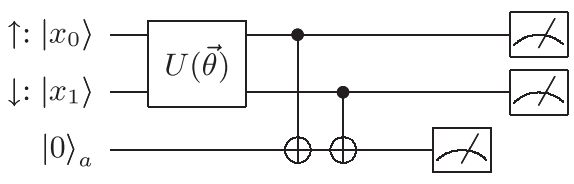

FIG. 1. A circuit to check the particle number parity of a physical trial state. The ancilla should be measured in $\left|\frac{1}{2}\left[1-(-1)^{N}\right]\right\rangle$. If errors occur, and the measured value of the ancilla is not correct, the measurement of the Hamiltonian term $\hat{h}_{i}$ on the register is not performed, and the circuit is reinitialized. 


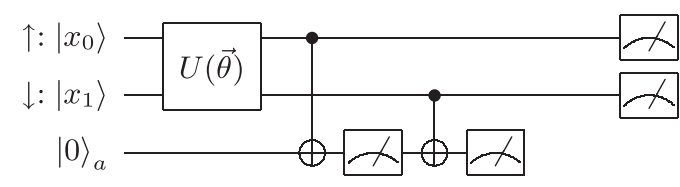

FIG. 2. A circuit to measure the spin parities. We compute the spin-up parity onto the ancilla and measure it. We then reset the ancilla to $|0\rangle$ and measure the spin-down parity.

If $N_{m}=0$ we measure the ancilla in $|+\rangle$, while if $N_{m}=1$ we measure the ancilla in $|-\rangle$. A worked example for $N=3$ is given in the Supplemental Material [31]. We can measure the spin numbers using a similar procedure.

The exact fraction of errors that can be detected will depend on the trial state produced by the Ansatz, but has a maximum value of $80 \%$ (see Supplemental Material [31]). In total, $M \log _{2}(N)$ control gates are needed to measure the electron number, or both spin numbers.

While our method can filter a fraction of the possible errors, the results will not be completely noise free. Consequently, we can combine our technique with other methods of error mitigation to further improve accuracy. The most straightforward approach is to use the detection method to filter out errors, and then use the extrapolation technique to obtain highly accurate results.

Hardware implementation.-We now consider how to implement our technique in current leading architectures, specifically trapped ion and superconducting systems. The circuit structure required is similar to that of a stabilizer evaluation for a topological code, which has been investigated for both platforms $[45,46]$. As can be seen from Fig. 1, the optimal implementation requires nonlocal gates. Trapped ion systems can perform gates between nonadjacent ions [47], so these circuits do not present any particular difficulty. Moreover, modular architectures are feasible designs for trapped ion systems [48,49]. Such architectures are networklike and could be constructed with the connectivity required for our circuits. As the coherence times of trapped ion qubits are considerably longer than their readout times [50], it is possible to carry out the checks using a single ancilla that is repeatedly measured and reinitialized.

In contrast, superconducting qubits typically have more limited connectivity, that may be nearest neighbor. With

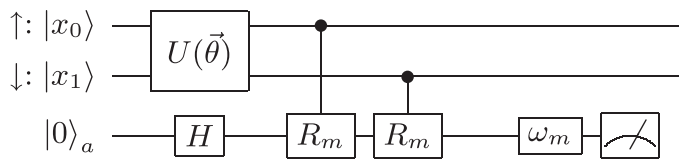

FIG. 3. The circuit that measures the $m$ th bit of the electron number $N_{m}$. The $R_{m}$ gates are given by $\operatorname{diag}\left(1, e^{\pi i / 2^{m-1}}\right)$. The gate $\omega_{m}$ is given by $\operatorname{diag}\left(1, e^{-\operatorname{dec}\left(N_{m-1} \ldots N_{1}\right) \pi i / 2^{m-1}}\right)$, where $\operatorname{dec}\left(N_{m-1} \ldots N_{1}\right)$ is the decimal representation of the binary string $N_{m-1} \ldots N_{1}$. We define $\omega_{1}$ as the identity matrix. Measurement of the ancilla is in the $X$ basis. such an architecture, we can implement our particle number check by using $O(M)$ SWAP gates to move the ancilla along the qubit register. It is possible to realize the parity checks with a shorter circuit, which was discussed in Ref. [51], and which we reproduce in the Supplemental Material [31]. In contrast, the number of gates required for a general UCC Ansatz is $O\left(M^{3}\right)$ [52]. For physical systems of interest, requiring $M=50-100$ qubits, the gate count will be dominated by the Ansatz circuit. Consequently, we expect the additional gates to have little impact on the detection rates derived above. For superconducting qubits, the measurement time can be of a comparable order of magnitude to the coherence time [53]. As such, it may be preferable to use multiple ancilla qubits, rather than to repeatedly reinitialize a single ancilla. This modest overhead constitutes two ancilla qubits for the spin number parity check and $\log _{2} N$ ancilla qubits for the spin number check.

Results.-We tested our method's efficacy in a VQE calculation on the simplest model of $\mathrm{H}_{2}$, with two electrons in four spin orbitals. We used a spin-conserving UCC Ansatz applied to the Hartree-Fock state. We did not consider the parameter update step of the VQE, so as to examine the effect of errors without consideration of a classical optimization algorithm [17]. Numerical simulations were performed using QUEST [54], and the simulation code can be found in Ref. [55]. Our aim was to measure the energy to within "chemical accuracy" (1.6 mhartree), which enables the prediction of reaction rates to within an order of magnitude at room temperature.

To detect errors, we performed error-prone checks of both the spin-up and spin-down parity numbers, using the circuit shown in the Supplemental Material [31]. This circuit has nearest-neighbor connectivity, and so lower bounds the efficacy of our method. We designed our simulations to mimic the actions of an experimentalist; the expectation value of each term in the Hamiltonian was found by repeating the circuit and measurement procedure many times. The number of measurements used is discussed in the Supplemental Material [31].

Initially, we considered energy measurements on a trial state that contained all four possible vectors, as described by Eq. (1). We used a symmetric depolarizing noise model, and set the 2-qubit gate error rate to be 10 times larger than the single-qubit gate error rate. We measured the energy of the state prepared by the Ansatz under the following conditions: (1) no parity check, errors, no extrapolation; (2) no parity check, errors, extrapolation; (3) parity check, errors, no extrapolation; (4) parity check, errors, extrapolation. We compare these results to the true energy extracted in the limit of infinite measurements and no gate errors. The results are shown in Fig. 4, using 2-qubit gate error rates ranging from $0.1 \%$ to $2 \%$. There were 92 single-qubit gates and 562 -qubit gates in the UCC Ansatz circuit-which we can approximate as 65 2-qubit gates. 


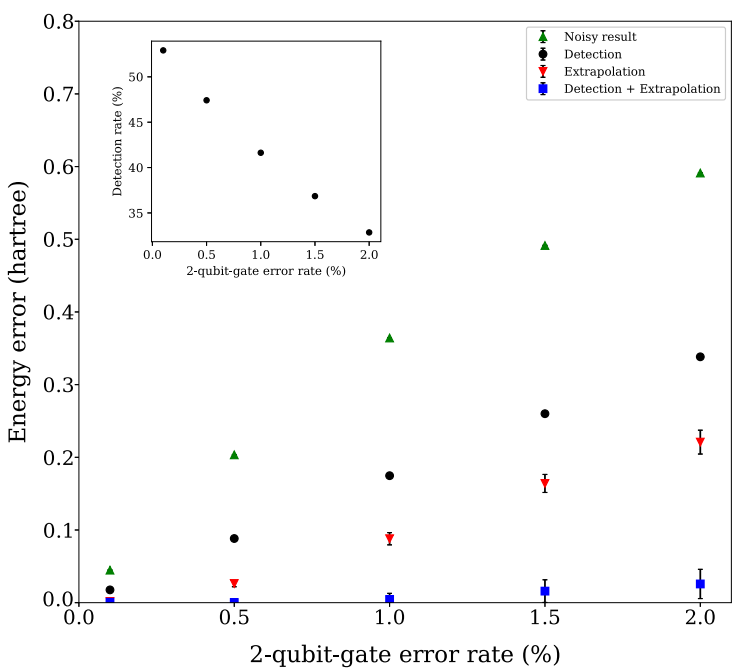

FIG. 4. Comparing methods of mitigating errors in simulations of $\mathrm{H}_{2}$. The detection rates shown in the inset were obtained from the numerical simulations. The true energy value was -1.1227 hartree. The error bars upper bound the standard error in the result.

The parity checks contributed an additional eight errorprone 2-qubit gates.

We see from Fig. 4 that the error detection method alone improves the accuracy of our energy measurements. While detection is less effective than extrapolation, a greater benefit can be obtained by combining the two methods. The accuracy of the combined method does not worsen significantly as the error rate increases, unlike the two individual methods. We observe from the inset plot that the fraction of detected errors falls approximately linearly with increasing error rate. When the error rate is small we detect around $53 \%$ of the errors. If we assume that we can only detect errors which occur during the Ansatz circuit (due to the use of nearest-neighbor gates for the parity checks), then our detection probability is given by the probability of an error happening in the Ansatz circuit (65/73) multiplied by the probability of detection $(10 / 15)$, which is roughly $59 \%$. We attribute the deviation of our result from this value to the use of a UCC Ansatz, which enables errors to propagate. At higher error rates, multiple errors are able to occur in the circuit, which reduces the fraction of errors that we can detect to close to one third.

We also used our method when calculating the dissociation curve of $\mathrm{H}_{2}$. We compare the true energy values with noisy measurements without error mitigation, noisy measurements with extrapolation, and noisy measurements with detection and extrapolation. The 2-qubit depolarizing error rate was $0.1 \%$, which has been achieved in a controlled setting [20,21], and should be targeted in near-future quantum computers. We combined this with temporally correlated over or under rotations of up to $1 \%$ (see Supplemental Material [31]). While previous experimental VQE calculations on $\mathrm{H}_{2}$ have achieved accurate

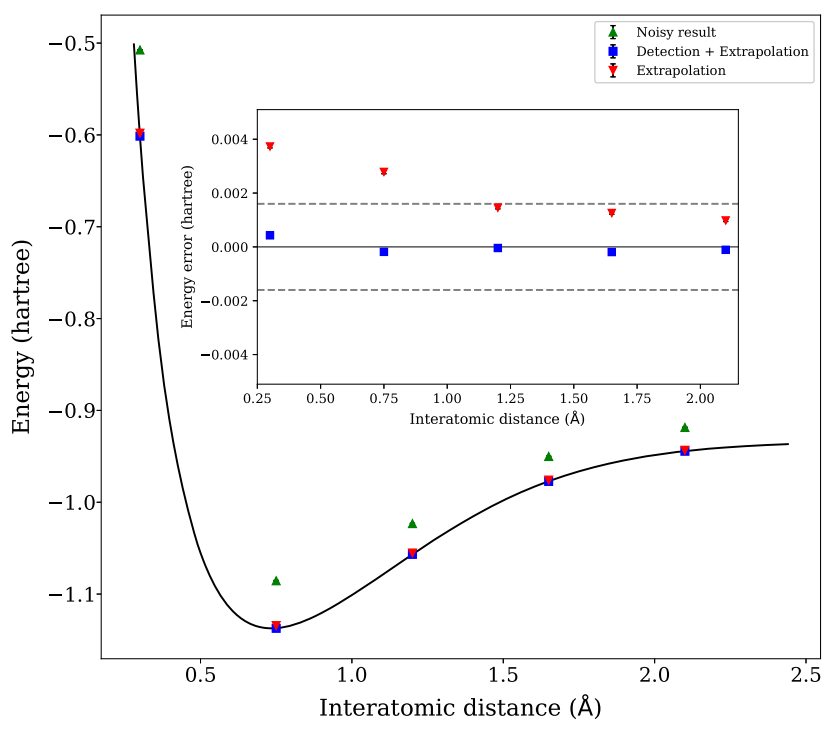

FIG. 5. Comparing methods of mitigating errors in simulations of $\mathrm{H}_{2}$. The error model is described in the main text. The inset shows the residual from the true value. The dashed lines in the inset mark chemical accuracy ( \pm 1.6 mhartree). The error bars show the standard error in the result, given in Ref. [31].

results with higher error rates [14-16,26,27,56], these experiments used fewer gates (and often fewer qubits) obtained using simplifications that are applicable to $\mathrm{H}_{2}$, but not to larger molecules. We chose to use the nonoptimized circuits to ensure that our simulations were representative of general chemistry problems.

We see from Fig. 5 that our method can obtain chemically accurate energies, even when the results would otherwise be corrupted by noise. The combined mitigation method achieves quantitatively accurate results, with a mean absolute residual of 0.2 mhartree. Compared to the unmitigated results, the deviation from the true value is reduced by a median factor of 239 (with a range of 141-818 and a mean of 340) by combining the error mitigation techniques. Compared to the extrapolated results, the deviation from the true value is reduced by a median factor of 9.1 (with a range of 6.6-35.6 and a mean of 15.0) by combining the two mitigation methods. As energies are exponentiated when calculating reaction rates, this improvement will be magnified when performing calculations of interest.

Discussion.-We have introduced a method to mitigate errors in near-term digital quantum simulations, which requires minimal additional resources. Our technique can be used to detect errors which change conserved quantities. It can be applied to calculations of both static and dynamical properties.

Our method can improve the accuracy of variational calculations, especially when combined with the extrapolation method of error mitigation. We simulated a noisy VQE calculation of the hydrogen molecule, and found that using this approach reduced the deviation from the true 
result by 2 orders of magnitude. Our simulations were performed with nearest-neighbor connectivity, showing the method's practicality.

Recent work has shown that surpassing classical simulation techniques with non-error-corrected quantum computers will likely require at least $10^{4}$ gates $[52,57,58]$. However, it is difficult to foresee error rates below $0.01 \%$ at which point we would expect an error to occur in every circuit, on average. Error mitigation techniques, such as those presented herein, may enable us to extract meaningful results from these simulations, providing a practical use for near-term quantum hardware. Consequently, implementing these techniques on recently announced devices [59-62]which will be cloud accessible, have 2-qubit-gate fidelities approaching $99 \%$, and possess tens of qubits-would provide an interesting avenue for future study.

While our method can detect a large proportion of errors, additional mechanisms will be required to provide error resilience for long circuits on non-error-corrected machines. One possibility is to combine our method with a 2-qubit phase-error detection code. Alternatively, one could utilize other invariant quantities. It was noted in Ref. [63] that the Hamiltonians of small molecules contain several symmetries. Evolution under a Hamiltonian variational Ansatz may conserve these quantities, enabling the design of additional checks. Future work will investigate the performance of concatenated mitigation methods for larger problems [31-38].

We thank S. Endo for insightful discussions. This work was supported by BP plc and the EPSRC National Quantum Technology Hub in Networked Quantum Information Technology (EP/M013243/1). We acknowledge the use of the University of Oxford Advanced Research Computing facility [64].

Note added.-Recently, a relevant work was posted by Bonet-Monroig et al. [51]. They suggest a similar method of error mitigation, and an elegant method for error mitigation via postprocessing. While their results focus on comparing their techniques, our results are consistent and can be compared.

sam.mcardle.science@gmail.com

†xiao.yuan.ph@gmail.com

simon.benjamin@materials.ox.ac.uk

[1] P. Dirac, Proc. R. Soc. A 123, 714 (1929).

[2] R. P. Feynman, Int. J. Theor. Phys. 21, 467 (1982).

[3] S. Lloyd, Science 273, 1073 (1996).

[4] A. Aspuru-Guzik, A. D. Dutoi, P. J. Love, and M. HeadGordon, Science 309, 1704 (2005).

[5] R. Babbush, N. Wiebe, J. McClean, J. McClain, H. Neven, and Garnet Kin-Lic Chan, Phys. Rev. X 8, 011044 (2018).

[6] A. Aspuru-Guzik, R. Lindh, and M. Reiher, ACS Cent. Sci. 4, 144 (2018).
[7] M. Reiher, N. Wiebe, K. M. Svore, D. Wecker, and M. Troyer, Proc. Natl. Acad. Sci. U.S.A. 114, 7555 (2017).

[8] I. D. Kivlichan, C. Gidney, D. W. Berry, N. Wiebe, J. McClean, W. Sun, Z. Jiang, N. Rubin, A. Fowler, A. Aspuru-Guzik, R. Babbush, and H. Neven, arXiv:1902.10673.

[9] R. Babbush, C. Gidney, D. W. Berry, N. Wiebe, J. McClean, A. Paler, A. Fowler, and H. Neven, Phys. Rev. X 8, 041015 (2018).

[10] A. Peruzzo, J. McClean, P. Shadbolt, M.-H. Yung, X.-Q. Zhou, P. J. Love, A. Aspuru-Guzik, and J. L. OBrien, Nat. Commun. 5, 4213 (2014).

[11] M.-H. Yung, J. Casanova, A. Mezzacapo, J. McClean, L. Lamata, A. Aspuru-Guzik, and E. Solano, Sci. Rep. 4, 3589 (2014).

[12] J. R. McClean, J. Romero, R. Babbush, and A. AspuruGuzik, New J. Phys. 18, 023023 (2016).

[13] D. Wecker, M. B. Hastings, and M. Troyer, Phys. Rev. A 92 , 042303 (2015).

[14] P. J. J. O’Malley et al., Phys. Rev. X 6, 031007 (2016).

[15] A. Kandala, A. Mezzacapo, K. Temme, M. Takita, M. Brink, J. M. Chow, and J. M. Gambetta, Nature (London) 549, 242 (2017).

[16] C. Hempel, C. Maier, J. Romero, J. McClean, T. Monz, H. Shen, P. Jurcevic, B. P. Lanyon, P. Love, R. Babbush, A. Aspuru-Guzik, R. Blatt, and C. F. Roos, Phys. Rev. X 8, 031022 (2018).

[17] N. P. D. Sawaya, M. Smelyanskiy, J. R. McClean, and A. Aspuru-Guzik, J. Chem. Theory Comput. 12, 3097 (2016).

[18] E. T. Campbell, B. M. Terhal, and C. Vuillot, Nature (London) 549, 172 (2017).

[19] C. Ballance, T. Harty, N. Linke, and D. Lucas, arXiv: 1406.5473 .

[20] C. J. Ballance, T. P. Harty, N. M. Linke, M. A. Sepiol, and D. M. Lucas, Phys. Rev. Lett. 117, 060504 (2016).

[21] J. P. Gaebler, T. R. Tan, Y. Lin, Y. Wan, R. Bowler, A. C. Keith, S. Glancy, K. Coakley, E. Knill, D. Leibfried, and D. J. Wineland, Phys. Rev. Lett. 117, 060505 (2016).

[22] Y. Li and S.C. Benjamin, Phys. Rev. X 7, 021050 (2017).

[23] K. Temme, S. Bravyi, and J. M. Gambetta, Phys. Rev. Lett. 119, 180509 (2017).

[24] S. Endo, S. C. Benjamin, and Y. Li, Phys. Rev. X 8, 031027 (2018).

[25] J. R. McClean, M. E. Kimchi-Schwartz, J. Carter, and W. A. de Jong, Phys. Rev. A 95, 042308 (2017).

[26] A. Kandala, K. Temme, A. D. Córcoles, A. Mezzacapo, J. M. Chow, and J. M. Gambetta, Nature (London) 567, 491 (2019).

[27] J. I. Colless, V. V. Ramasesh, D. Dahlen, M. S. Blok, M. E. Kimchi-Schwartz, J. R. McClean, J. Carter, W. A. de Jong, and I. Siddiqi, Phys. Rev. X 8, 011021 (2018).

[28] C. Song, J. Cui, H. Wang, J. Hao, H. Feng, and Y. Li, arXiv: 1812.10903.

[29] I. G. Ryabinkin and S. N. Genin, arXiv:1812.09812.

[30] I. G. Ryabinkin, S. N. Genin, and A. F. Izmaylov, J. Chem. Theory Comput. 15, 249 (2019).

[31] See Supplemental Material at http://link.aps.org/ supplemental/10.1103/PhysRevLett.122.180501 for derivation of detection rates, and details of the numerical simulations performed, which includes Refs. [32-38]. 
[32] J. T. Seeley, M. J. Richard, and P. J. Love, J. Chem. Phys. 137, 224109 (2012).

[33] M. A. Nielsen and I. L. Chuang, Quantum Computation and Quantum Information (Cambridge University Press, Cambridge, 2002).

[34] A. K. Ekert, C. M. Alves, D. K. L. Oi, M. Horodecki, P. Horodecki, and L. C. Kwek, Phys. Rev. Lett. 88, 217901 (2002).

[35] A. Y. Kitaev, arXiv:quant-ph/9511026.

[36] J. R. McClean et al., arXiv:1710.07629.

[37] J. D. Whitfield, J. Biamonte, and A. Aspuru-Guzik, Mol. Phys. 109, 735 (2011).

[38] I. G. Hughes and T. P. A. Hase, Measurements and Their Uncertainties (Oxford University Press, Oxford, 2010).

[39] J. R. McClean, S. Boixo, V. N. Smelyanskiy, R. Babbush, and H. Neven, Nat. Commun. 9, 4812 (2018).

[40] P. K. Barkoutsos, J. F. Gonthier, I. Sokolov, N. Moll, G. Salis, A. Fuhrer, M. Ganzhorn, D. J. Egger, M. Troyer, A. Mezzacapo, S. Filipp, and I. Tavernelli, Phys. Rev. A 98, 022322 (2018).

[41] D. Gottesman, arXiv:quant-ph/9705052.

[42] J. Romero, R. Babbush, J. R. McClean, C. Hempel, P. J. Love, and A. Aspuru-Guzik, Quantum Sci. Technol. 4, 014008 (2019).

[43] J. Preskill, Quantum 2, 79 (2018).

[44] S. McArdle, T. Jones, S. Endo, Y. Li, S. Benjamin, and X. Yuan, arXiv:1804.03023.

[45] A. Bermudez, X. Xu, R. Nigmatullin, J. O'Gorman, V. Negnevitsky, P. Schindler, T. Monz, U. G. Poschinger, C. Hempel, J. Home, F. Schmidt-Kaler, M. Biercuk, R. Blatt, S. Benjamin, and M. Müller, Phys. Rev. X 7, 041061 (2017).

[46] A. G. Fowler, M. Mariantoni, J. M. Martinis, and A. N. Cleland, Phys. Rev. A 86, 032324 (2012).

[47] N. M. Linke, D. Maslov, M. Roetteler, S. Debnath, C. Figgatt, K. A. Landsman, K. Wright, and C. Monroe, Proc. Natl. Acad. Sci. U.S.A. 114, 3305 (2017).
[48] C. Monroe, R. Raussendorf, A. Ruthven, K. R. Brown, P. Maunz, L.-M. Duan, and J. Kim, Phys. Rev. A 89, 022317 (2014).

[49] N. H. Nickerson, J. F. Fitzsimons, and S. C. Benjamin, Phys. Rev. X 4, 041041 (2014).

[50] R. Noek, G. Vrijsen, D. Gaultney, E. Mount, T. Kim, P. Maunz, and J. Kim, Opt. Lett. 38, 4735 (2013).

[51] X. Bonet-Monroig, R. Sagastizabal, M. Singh, and T. E. O’Brien, Phys. Rev. A 98, 062339 (2018).

[52] M. Motta, E. Ye, J. R. McClean, Z. Li, A. J. Minnich, R. Babbush, and G. K.-L. Chan, arXiv:1808.02625.

[53] G. Wendin, Rep. Prog. Phys. 80, 106001 (2017).

[54] T. Jones, A. Brown, I. Bush, and S. Benjamin, arXiv:1802 .08032 .

[55] S. McArdle, Github repository, https://github.com/ sammcardle 30 .

[56] M. Ganzhorn, D. J. Egger, P. K. Barkoutsos, P. Ollitrault, G. Salis, N. Moll, A. Fuhrer, P. Mueller, S. Woerner, I. Tavernelli, and S. Filipp, arXiv:1809.05057.

[57] Z. Jiang, K. J. Sung, K. Kechedzhi, V. N. Smelyanskiy, and S. Boixo, Phys. Rev. Applied 9, 044036 (2018).

[58] S. McArdle, S. Endo, A. Aspuru-Guzik, S. Benjamin, and X. Yuan, arXiv:1808.10402.

[59] K. Wright et al., arXiv:1903.08181.

[60] A preview of Bristlecone, Google's new quantum processor (2018), https://ai.googleblog.com/2018/03/a-preview-ofbristlecone-googles-new.html.

[61] Cramming more power into a quantum device (2019), https://www.ibm.com/blogs/research/2019/03/powerquantum-device/.

[62] A. Nersisyan, S. Poletto, N. Alidoust, R. Manenti, R. Renzas, C.-V. Bui, K. Vu, T. Whyland, Y. Mohan, E. A. Sete, S. Stanwyck, A. Bestwick, and M. Reagor, arXiv:1901 .08042 .

[63] S. Bravyi, J. M. Gambetta, A. Mezzacapo, and K. Temme, arXiv: 1701.08213

[64] http://dx.doi.org/10.5281/zenodo.22558. 